$\underline{\text { Research Article }}$

\title{
Knowledge, attitude and practices towards dengue among undergraduate students at a University in Sri Lanka
}

\author{
NGN Gayathri, PDNN Sirisena, UTN Senaratne, F Noordeen \\ Sri Lankan Journal of Infectious Diseases 2021 Vol.11(2):73-83 \\ DOI: http://dx.doi.org/10.4038/sljid.v11i2.8407
}

\begin{abstract}
Background: The present study was undertaken to determine knowledge, attitude and practices (KAP) regarding dengue among undergraduate students at the University of Peradeniya, which is the largest university in Sri Lanka.

Methods: A total of 384 randomly selected students from 9 different faculties, including 191 females and 193 males were surveyed. A pre-tested questionnaire was used to assess the KAP and the analysis scale for KAP sections ranged from 'satisfactory', 'fair' and 'poor'.

Results: A higher percentage (40-60\%) of undergraduate students from Dental, Medical, and Veterinary faculties had satisfactory knowledge status while $<21 \%$ of the undergraduates from other faculties had obtained a satisfactory knowledge score indicating a significant difference $(p$ $<0.05)$ between health related and non-health related faculties regarding knowledge on dengue. The attitude score also significantly differed among the faculties $(p=0.001)$. Knowledge level on causation, signs and symptoms of dengue, complications, identifying the correct peak times in the year, identifying the vectors and the breeding sites was satisfactory among students from the health-related faculties. In general, attitude level was satisfactory with more than $75 \%$ of the study population giving correct answers for each of the question under that category. Practices regarding the disposal of solid waste and updated knowledge on solid waste management were also satisfactory.
\end{abstract}

Conclusion: Overall, 59.9\% of the study participants reached fair to satisfactory knowledge scores and $60.9 \%$ of the study participants reached fair to satisfactory practice scores. High attitude levels indicate the willingness to participate and contribute to reduce the dengue burden. Minimizing the gaps in KAP of the undergraduates should be encouraged through targeted awareness programmes.

Department of Microbiology, Faculty of Medicine, University of Peradeniya, Sri Lanka

Address for correspondence: Prof F. Noordeen, Department of Microbiology, Faculty of Medicine, University of Peradeniya, Sri Lanka Telephone: +0094772293301/81 2396530

E-mail: faseeha.noordeen12@gmail.com; faseehan@pdn.ac.lk

https://orcid.org/0000-0002-2018-0606

Received 28 June 2021 and revised version accepted 3 October 2021.

C) (†) use, distribution, and reproduction in any medium, provided the original author and source are credited. 
Keywords: Dengue, knowledge, attitude, practice, university students

\section{Introduction}

Dengue is a viral infection caused by the dengue virus (DENV) of the family Flaviviridae and genus Flavivirus. ${ }^{1}$ The virus is transmitted by the mosquitoes Aedes aegypti and Aedes albopictus and has four serotypes DENV-1, DENV-2, DENV-3, and DENV-4. ${ }^{1,2}$ Many patients who are infected with DENV remain asymptomatic. Some develop symptomatic DENV infection. which might result in severe dengue. ${ }^{1,2,3}$ High fever, severe headache, flushing, myalgia and arthralgia, vomiting and acute abdominal pain are the general features of dengue. Bleeding manifestations and plasma leakage occur in severe dengue. ${ }^{1,2,3}$ Annually, 50 million people are infected with DENV of whom 50,000 suffer severe dengue. ${ }^{4}$

The first reported dengue case in Sri Lanka was in 1962 with the first outbreak from 1965 to 1966. ${ }^{4}$ The first major dengue epidemic was reported in the country in 1989 and since 1996, dengue has been a notifiable disease in Sri Lanka. Between 2010 and 2019, several dengue epidemics have occurred, and the number of cases is increasing every year with 175,644 cases reported in 2018. ${ }^{5}$ Dengue has become a major public health hurdle in Sri Lanka in the past four decades. Despite strict vector control measures and updated triage protocols for diagnosis and management of dengue, the number of cases keeps on increasing.

Prevention and control of dengue depend mainly on minimizing transmission of the virus and vector control. ${ }^{6,7}$ On the other hand, the epidemiology and ecology of dengue is linked to human behaviour. Hence, employing integrated control measures such as improving knowledge, attitude, and practices (KAP) related to dengue in different sectors of the public including university students is important. ${ }^{8}$ In conjunction with better KAP in different sectors of the public including university students, vector control can be improved in order to reduce the dengue burden in the country. Public awareness and contribution of the public in mosquito control and environmental management is crucially important. It is therefore a necessity to assess KAP levels on dengue to decide how to improve public education, starting from educated youth such as university undergraduates who are the next leaders of a country. Evaluating the KAP levels of undergraduates will be useful to identify gaps which need to be corrected and help promote self and social responsibility in reducing the dengue burden in the country.

The current study mainly focused on assessing KAP on dengue and its prevention and control among undergraduate students at the University of Peradeniya. Studies have been conducted in other countries as well as in Sri Lanka among various populations including school children and the general public. However, no data is available on KAP among university students who are at the tertiary level of education and are placed in a better position to contribute to dengue control activities. 


\section{Methods}

\section{Study design, study sample and data collection}

This was a prospective descriptive study designed to assess KAP on dengue among undergraduate students at the University of Peradeniya. The data were collected from randomly selected students $(n=384$; Age $=20-30$ years) from all faculties $(n=9)$, namely Agriculture, Arts, Allied Health Sciences (AHS), Dental Sciences, Engineering, Management, Medicine, Science and Veterinary Medicine for 3 months from May to July 2016.

A self-administered questionnaire was validated using a pilot study undertaken among undergraduate students from the same university and was modified after pre-testing. The volunteers for the pilot study were excluded from the main study. The questionnaire consisted of questions on socio-demographic details (age, gender, ethnicity, residence, faculty, parents' educational background and occupation), knowledge on dengue (disease signs and symptoms, complications, vectors, breeding sites and incubation period), attitude and practices (control and prevention) towards dengue. The questionnaire comprised of both defined (Yes/No) and openended questions.

\section{Statistical analysis}

\section{Analysis of knowledge}

A cumulative knowledge score (CKS) was calculated as a total of all knowledge-based questions. A total of 9 questions with sub sections assessing knowledge on dengue were given 18 marks. For each of the correct answers, +1 was given. For a wrong answer or if the answer was not known 0 marks was given. For questions with multiple correct answers, one point was given for each of the correct answers. The total knowledge score ranged from 0 to 18. The scale was classified as follows: 'satisfactory' >mean+SD; 'fair' =mean-SD to mean+SD' and 'poor'= <mean-SD (mean 8.88, SD 1.42).

\section{Analysis of attitude}

A total of 7 questions were used to assess the attitude towards dengue. The participants were assessed on the attitude on management and prevention measures.

\section{Analysis of practices}

A total of 7 questions with sub sections, including two open ended questions, were used to assess practices towards dengue with 13 marks. For each correct answer +1 , incorrect answer -1 and for no response 0 marks were given. The total score ranged from 0 to 13 . For the questions with multiple correct answers, one point was given for each of the correct answers. The scale was classified as follows: 'satisfactory' $>$ mean $+\mathrm{SD}$, 'fair' $=$ mean to mean $+\mathrm{SD}$ and 'poor' $=<$ mean (mean 4.7, SD 0.97).

The data was analyzed using the SPSS software version 20. Student's $t$ test was used to calculate the association between each KAP score and age and gender. Analysis of variance (ANOVA) was performed to determine the association between each KAP level and the faculty. The association between the KAP scores for health-related faculties (AHS, Dental Sciences, Medicine and Veterinary Medicine) and non-health related faculties (Agriculture, Arts, 
Engineering, Management and Science) was analyzed using the Student's $t$ test and $p<0.05$ was considered as statistically significant.

\section{Results}

The socio-demographic characteristics of the study participants were diverse as given in Table 1 .

Table 1: Summary of socio-demographic characteristics of the study participants ( $\mathrm{n}=384)$.

\begin{tabular}{|l|c|c|}
\hline $\begin{array}{c}\text { Sample } \\
\text { characteristics }\end{array}$ & $\begin{array}{c}\text { No. } \\
\text { representing } \\
\text { each } \\
\text { category }\end{array}$ & $\begin{array}{c}\text { Percentage } \\
\text { (\%) }\end{array}$ \\
\hline Gender \\
\hline Male \\
\hline Female \\
\hline Age group & 193 & 50.3 \\
\hline 20-25 years & 354 & 49.7 \\
\hline 26-30 years & 30 & 92.2 \\
\hline Ethnicity & 308 & 80.9 \\
\hline Sinhala & 42 & 10.9 \\
\hline Tamil & 34 & 8.2 \\
\hline Muslim
\end{tabular}

KAP scores followed a normal distribution, hence, parametric tests were applied for statistical analysis. A significant difference $(p=0.02)$ was noted in the knowledge score between the two age groups and a significant difference was noted between the practices between males and females $(p=0.001)$. The knowledge score and the attitude score significantly differed among different faculties $(p=0.001)$ (Table 2 and Table 3). Moreover, when the faculties were divided as health related and non-health related faculties, a significant difference was observed in the knowledge score $(p=0.001)$.

T able 2: KAP score of the study participants against demogaphic data.

\begin{tabular}{|c|c|c|c|c|c|c|}
\hline Variable & $\begin{array}{l}\text { Knowledge } \\
\text { score } \\
\text { (Mean } \pm \text { SD) }\end{array}$ & $\underset{\text { value }}{p}$ & $\begin{array}{c}\text { Attitude } \\
\text { score } \\
\text { (Mean } \pm \text { SD) }\end{array}$ & $\underset{\text { value }}{p}$ & $\begin{array}{c}\text { Practice } \\
\text { score } \\
(\text { Mean } \pm S D)\end{array}$ & $\underset{\text { value }}{p}$ \\
\hline \multicolumn{7}{|l|}{ Age group } \\
\hline $20-25$ & $8.84 \pm 1.41$ & \multirow[t]{2}{*}{$0.02 *$} & $6.54 \pm 0.81$ & \multirow[t]{2}{*}{$0.25^{*}$} & $4.69 \pm 1.03$ & \multirow[t]{2}{*}{$0.60^{*}$} \\
\hline $25-30$ & $9.47 \pm 1.44$ & & $6.36 \pm 0.76$ & & $4.8 \pm 0.92$ & \\
\hline \multicolumn{7}{|l|}{ Gender } \\
\hline Male & $8.81 \pm 1.42$ & \multirow[t]{2}{*}{$0.27^{*}$} & $6.51 \pm 0.91$ & \multirow[t]{2}{*}{$0.79^{*}$} & $4.5 \pm 1.07$ & \multirow[t]{2}{*}{$0.001 *$} \\
\hline Female & $8.97 \pm 1.42$ & & $6.54 \pm 0.69$ & & $4.91 \pm 0.93$ & \\
\hline \multicolumn{7}{|l|}{ Faculty } \\
\hline Agriculture & $8.58 \pm 1.26$ & \multirow[t]{9}{*}{$0.001^{\#}$} & $6.13 \pm 0.92$ & \multirow[t]{9}{*}{$0.04^{\#}$} & $4.93 \pm 1.12$ & \multirow[t]{9}{*}{$0.001^{\#}$} \\
\hline $\mathrm{AHS}$ & $9.69 \pm 1.87$ & & $6.61 \pm 0.57$ & & $4.26 \pm 0.96$ & \\
\hline Arts & $8.54 \pm 1.32$ & & $6.58 \pm 0.85$ & & $4.80 \pm 0.96$ & \\
\hline Dental & $9.20 \pm 1.27$ & & $6.67 \pm 0.72$ & & $4.20 \pm 1.01$ & \\
\hline Engineering & $8.98 \pm 1.31$ & & $6.40 \pm 0.95$ & & $4.71 \pm 1.00$ & \\
\hline Management & $8.39 \pm 0.98$ & & $6.50 \pm 0.92$ & & $4.72 \pm 0.95$ & \\
\hline Medicine & $9.55 \pm 1.34$ & & $6.72 \pm 0.56$ & & $5.27 \pm 0.74$ & \\
\hline Science & $8.92 \pm 1.48$ & & $6.61 \pm 0.71$ & & $4.52 \pm 1.16$ & \\
\hline Veterinary & $9.28 \pm 1.44$ & & $6.14 \pm 0.66$ & & $4.00 \pm 0.55$ & \\
\hline
\end{tabular}

'Significance value based on Student's t test; "Sigrificance value based on analysis of variance (ANOVA). 
Table 3: Post hoc analysis of based on analy sis of variance (ANOVA) KAP score

\begin{tabular}{|l|c|c|c|}
\hline $\begin{array}{l}\text { Type of } \\
\text { the score }\end{array}$ & $\begin{array}{c}\text { Comparison between } \\
\text { faculties }\end{array}$ & $\begin{array}{c}\boldsymbol{p} \\
\text { value }\end{array}$ \\
\hline Knowledge & AHS & Arts & 0.004 \\
\hline & Medicine & Arts & 0.007 \\
\hline Attitude & AHS & Arts & 0.05 \\
\hline & Medicine & Arts & 0.001 \\
\hline Fractice & AHS & Medicine & 0.0003 \\
\hline & Dental & Medicine & 0.013 \\
\hline & Medicine & Science & 0.009 \\
\hline & Medicine & Veterinary & 0.002 \\
\hline
\end{tabular}

No statistically significant difference in knowledge $(p=0.65)$, attitude $(p=0.55)$ and practice $(p=0.08)$ were noted between the participants who had dengue and those who did not have dengue. Overall, $15.9 \%$ of the participants had satisfactory and $44 \%$ of the participants had fair and $40.1 \%$ of the participants had poor knowledge scores for dengue disease including the seasonality, vector and dengue control related questions.

\section{Knowledge on dengue among the study participants}

Knowledge on dengue and its signs and symptoms, complications, seasonality, mosquito vector, biting time, breeding sites and home remedies used were analyzed. Of the 384 undergraduates, $324(85.2 \%)$ knew that dengue is a viral disease. Only $66(17.1 \%)$ knew about the dengue seasonality in the country and that there are two peaks, November-February, and JuneSeptember. A total of 221 (57.4\%) undergraduates knew that dengue is a mosquito transmitted disease. Furthermore, no correlation was noted between participants' knowledge scores and parental educational level or occupation $(p>0.05)$.

Table 4: Attitude of the study participants towards dengue / dengue contr ol.

\begin{tabular}{|l|c|c|}
\hline Attitude & Number (n) & Per centage (\%) \\
\hline I am aware about the dengue risk \\
\hline Yes & 351 & 91.4 \\
\hline No & 33 & 8.6 \\
\hline I am satisfied with the knowl edge on preventive measures \\
\hline Yes & 293 & 76.3 \\
\hline No & 91 & 23.7 \\
\hline I am aware that dengue is a recurrent disease \\
\hline Yes & 355 & 92.5 \\
\hline No & 29 & 7.6 \\
\hline $\begin{array}{l}\text { I agree with the need for frequently checking for breeding } \\
\text { sites }\end{array}$ \\
\hline Yes & 356 & 92.7 \\
\hline No & 28 & 7.3 \\
\hline I agree that dengue affects all age groups \\
\hline Yes & 371 & 96.6 \\
\hline No & 13 & 3.4 \\
\hline When I suspect dengue fever \\
\hline Will see a doctor & 379 & 98.7 \\
\hline Buy medicines myself & 5 & 1.3 \\
\hline Who is responsible for the mosquito control activities \\
\hline Government & 376 & 97.9 \\
\hline Individuals & 7 & 1.8 \\
\hline Community & 1 & 0.3 \\
\hline All of above & 0 \\
\hline
\end{tabular}

\section{Attitude towards dengue}

The responses for attitude through different questions on dengue and dengue control are given Table 4.

\section{Practices towards dengue}

Of the 384 participants, $274 \quad(71.2 \%)$ answered that they would see a doctor in the early phase of the illness, 27 (7\%) answered they would try home remedies first and $79(20.5 \%)$ responded positively to both options. For treating the patients at home, $246(63.9 \%)$ of the participants knew that adequate rest is required, 230 $(59.7 \%)$ thought the suspected patients must drink plenty of water/fluids, 184 (48.8\%) of participants preferred taking paracetamol to reduce fever and 27 (7\%) said that they would take aspirin, 30 $(7.8 \%)$ of the participants did not know which of these drugs to be taken (Table 5). 
Table 5: Practices of the study participants towards dengue/ dengue control.

\begin{tabular}{|l|c|c|}
\hline Questions & Number (n) & Percentage (\%) \\
\hline What will you do in the early p hase of the fev er? & 27 & 7.0 \\
I will get some hom e remedies & 274 & 71.2 \\
I will see a doctor & 79 & 20.5 \\
Both & 0 & 0.0 \\
\hline Any other & 184 & 47.8 \\
\hline How will you manage a susp ected patient at home? & 27 & 7.0 \\
Reduce fever by taking paracetam ol & 230 & 59.7 \\
Give aspirin & 246 & 63.9 \\
Give adequate water/ fluids & 30 & 7.8 \\
Allow to rest & 2 & 0.5 \\
\hline Do not know & & \\
Any other & 320 & 83.1 \\
\hline How do youp revent mosq uito b ites? & 268 & 69.6 \\
By keeping the environment clean & 141 & 36.6 \\
By mosquito nets & 117 & 30.4 \\
By mosquito coils & 104 & 27.0 \\
By using repellents & 02 & 0.5 \\
By spr aying insecticides & & \\
Any other & 328 & 85.2 \\
\hline How do you eliminate mosquito breeding sites? & 261 & 67.8 \\
Proper removal of garbage/ dom estic waste & 209 & 54.3 \\
Add fish to ponds & 286 & 74.3 \\
Add salt to ant traps and standing water & 313 & 81.3 \\
Bury tyres, containers which can gather water & 04 & 1.0 \\
\hline Roof top clearing & & \\
Any other & 214 & 55.6 \\
\hline How do you disp ose solid waste? & 210 & 54.5 \\
Bury the waste & 23 & 5.8 \\
Burn the waste & 05 & 54.3 \\
Throw at the roadside & & 3.3 \\
\hline Separate and dispose in separ ate bins & 153 & 59.7 \\
Any other & 230 & 51.2 \\
\hline Have you ever contributed to mosquito control & 47.0 \\
programmes in your area? & & \\
Yes & & \\
No & & \\
\hline Do you have an up dated know ledge on dengue? & & \\
Yes & & \\
No & & \\
\hline
\end{tabular}

For questions on contribution to mosquito control activities in their areas, 230 (59.9\%) did not contribute to control activities in the past whereas $153(39.8 \%)$ answered that they had contributed to control activities in the past. Among the responders, 197 (51.2\%) answered that they had updated knowledge and $181(47 \%)$ answered that they did not have updated knowledge on dengue (Table 5). 


\section{Discussion}

Mean KAP scores of the 384 university students were compared with their demographic characteristics and a significant difference was noted in the mean knowledge score between age groups. Undergraduates of 26-30 years had greater scores indicating an age associated increment of knowledge compared to undergraduates of 20-25 years. However, a similar pattern was not observed for the attitude and the practice scores. A significantly higher practice score was noted for females. The knowledge and the attitude scores significantly differed among the faculties. When the faculties were divided into health related and non-health related, the health-related faculties had a significantly higher mean knowledge score (Table 2). Similar differences have also been reported elsewhere. ${ }^{9,10}$ The post hoc analysis revealed a significant difference between knowledge and attitude scores of students from AHS and Medical Faculties compared with those from the Arts Faculty. Furthermore, significant association was noted in the practice score among faculties (Table 3).

Similar to a few other studies conducted in Sri Lanka and other countries ${ }^{7,11,12}$ on KAP of dengue, most of the current responders were also aware of dengue. Moreover, the study participants were aware of signs and symptoms of dengue as noted by a previous study conducted in Colombo ${ }^{11}$. Many of the participants knew the incubation period of dengue but $40 \%$ of them did not know the reason for the incubation period. Many were aware of the peak seasons for dengue as June-September but only $17.1 \%$ knew that it occurs in NovemberFebruary and June-September with the monsoons. Knowledge regarding the seasonality of the disease will be useful to reduce the transmission of infection through vigilance and environmental management practices. Only 5.6\% of the current study participants knew that dengue mosquitoes breed in dirty water. This gap in knowledge must be improved among these students. Information on brackish water and septic water breeding of Aedes mosquitoes ${ }^{13-15}$ must be incorporated when dengue awareness programmes are organized for the public. We believe that updated knowledge will lead to improved attitude and practices towards dengue control.

Over fifty percent of the students from medicine (55.6\%) and veterinary medicine (64.2\%) faculties reached the satisfactory knowledge score. However, only $40 \%$ of dental students reached the satisfactory knowledge score. Less than $21 \%$ of students from other faculties reached the satisfactory knowledge score as was also noted in a study done in Saudi Arabia. ${ }^{7}$ In the current study, $57.7 \%$ of AHS students had a fair score for knowledge while $52.9 \%$ of the arts and $51.9 \%$ of the management students had poor scores. One would expect a higher percentage of the university students to have better mean scores for knowledge than what the study has produced. This is of great concern as a high percentage of students in tertiary learning do not have satisfactory knowledge for a common infection in the country.

More females had satisfactory scores for knowledge compared to males. More final year students had fair and satisfactory knowledge scores compared to their juniors. Past experience with dengue did not improve the KAP scores compared to disease naïve participants, contradicting the findings of the studies done in Taiwan ${ }^{16}$ and Pakistan ${ }^{17}$ where prior exposure has contributed to improve the knowledge level of their study participants. Moreover, parental education level and occupation did not improve the KAP scores, again negating a previous finding, which shows that parents' educational level plays a vital role in their children's KAP scores on dengue. ${ }^{7}$ 
In the current study, overall, 59.9\% of the participants had fair and satisfactory scores for knowledge, similar to the level reported previously. ${ }^{7}$ More than $50 \%$ of the participants had higher knowledge scores in a study conducted in Ecuador ${ }^{10}$ in a sample of healthcare workers. The overall mean knowledge scores in the current study $(8.81 \pm 1.42$ to $8.97 \pm 1.42)$ also reiterate that university and health authorities must be involved actively on health promotion activities to improve the knowledge levels of university students on dengue to promote a country with a minimum dengue burden.

The attitude towards dengue control was better in females than males. In general, attitude score was satisfactory for more than $75 \%$ of the participants. Majority of the participants were aware of the risk (91.4\%) and considered that control activities must be done by the government (97.9\%). Students at all the faculties were at fair to satisfactory level for attitude towards dengue and dengue control. Although many final year students have gained higher knowledge scores, they lacked the correct attitude towards dengue control. The attitude of undergraduates must be improved through awareness programmes highlighting the importance of social responsibility in controlling dengue rather than expecting it from the government. When taking the attitude towards dengue, there were similarities between this study and previous studies conducted in other countries ${ }^{7,17}$ and Sri Lanka. ${ }^{18}$ One would wonder why such an attitude of expecting the government to do dengue control activities prevails among the university students. University education must inculcate individual and social responsibilities among the students, the future leaders who would be tuned to get involve themselves in social activities like dengue awareness programmes.

Overall, only $12.5 \%$ of the participants had a satisfactory practice score. The low practice score for dengue control might reflect the increasing number of breeding sites. Good practices regarding the disposal of solid waste and updating knowledge can be considered as predictors of a satisfactory level for practice as in studies done elsewhere. ${ }^{7,19,20}$ Aspirin is a drug used to reduce fever when there are no haemorrhagic tendencies. Use of aspirin for controlling fever in dengue was not selected as an option by most of the current study participants (93\%) and suggests that participants are aware of the non-suitability of aspirin to relieve symptoms of dengue. In contrast, a previous study conducted in Colombo ${ }^{8}$ noted a significant number of their study participants selecting aspirin as an option to relieve symptoms of dengue. Regarding mosquito control, most of the undergraduates were aware that proper solid waste management, preventive measures for mosquito bites and eliminating breeding sites will reduce the disease burden. However, the willingness to participate or past participation in mosquito control activities was not satisfactory as more than $50 \%$ of the students did not contribute despite having updated knowledge on practices used to reduce DENV transmission. Poor participation in the past or a reduced willingness to participate in dengue control activities must be corrected through university education. The university training must emphasize the importance of contributing to common goals like reducing the dengue burden in the country.

The study consisted of a random sample from the University of Peradeniya, and it is possible that the observations on the KAP on dengue might be different if the study was done among different sectors of the general public as the results Post hoc analysis showed. Future studies are needed to obtain KAP levels of the country to undertake more targeted control efforts to reduce the 
dengue burden.

\section{Conclusion}

In conclusion, $15.9 \%$ of the participants had satisfactory and $44 \%$ of the participants had fair and $40.1 \%$ of the participants had poor knowledge scores for dengue disease including the seasonality, vector and dengue control related questions. Gaps were identified in participants from certain faculties on KAP. Awareness programmes to promote attitudinal changes must be designed to improve KAP in the universities to help reduce the dengue related economic drain in the country.

\section{Declarations}

Acknowledgements: We thank Ms Shamla Najimudeen for checking the language and editing the manuscript.

Funding source: None.

Conflict of Interest statement: None declared.

Ethical approval for the study was obtained from the Ethical Review Committee of the Faculty of AHS, University of Peradeniya. Written informed consent was obtained from study participants prior to data collection.

NGNG carried out data collection and data analysis with PDNN Sirisena \& UTN Senaratne.

FN \& UTNS drafted the manuscript.

FN conceptualized the design of the study, critically read, improved and revised the manuscript.

All authors read and approved the final manuscript.

\section{References}

1. Senaratne TN, Noordeen F. Diagnosis of dengue in Sri Lanka: improvements to the existing state of the art in the island. Trans R Soc Trop Med Hyg. 2014;108: 685-691. doi: https://doi.org/10.1093/trstmh/tru131

2. Taksande A, Lakhkar B. Knowledge, attitude and practice (KAP) of dengue fever in the rural area of central India. Shiraz E-Med J. 1970; 13:146-157. No doi

3. Sirisena PDNN, Noordeen F. Evolution of dengue in Sri Lanka - changes in the virus, vector, and climate. Int J Infect Dis. 2014; 19:6-12. doi: https://doi.org/10.1016/j.ijid.2013.10.012

4. Vitarana T, Jayakuru WS, Withane N. Historical account to dengue haemorrhagic fever in Sri Lanka. WHO/SEARO. Dengue Bull. 1997; 21:117-118. No doi

5. Gideon Informatics, I. and S. Berger. Dengue. 2019; Available at http://public.eblib.com/choice/PublicFullRecord.aspx? $p=5723569$.

6. Sirisena PDNN, Noordeen F. Dengue control in Sri Lanka - improvements to the existing state of the art in the island. Sri Lankan J Infect Dis. 2016; 6: 2-16. doi: http://doi.org/10.4038/sljid.v6i1.8107

7. Ibrahim NK, Al-Bar A, Kordey M, et al. Knowledge, attitudes, and practices relating to Dengue fever among females in Jeddah high schools. J Infect Public Health. 2009;2:30-40.

doi: http://doi.org/10.1016/j.jiph.2009.01.004

8. Gunasekara TDCP, Velathanthiri VGNS, Weerasekara MM, et al. Knowledge, attitudes and practices regarding dengue fever in a sub urban community in Sri Lanka. Galle Med J. 2012; 17:10-17. doi: http://doi.org/10.4038/gmj.v17i1.4355

9. Ho TS, Huang MC, Wang SM, et al. Knowledge, attitude, and practice of dengue disease among healthcare professionals in southern Taiwan. J Formosan Med Assoc. 2013; 112:8-23. doi: http://doi.org/10.1016/j.jfma.2012.11.004

10. Handel AS, Ayala EB, Borbor-Cordova MJ, et al. Knowledge, attitudes, and practices regarding dengue infection among public sector healthcare providers in Machala, Ecuador. Tropical diseases, Travel Medicine and Vaccines. 2016; 2:8. doi: http://doi.org/10.1186/s40794-016-0024-y

11. Nalongsack S, Yoshida Y, Morita M, et al. Knowledge, attitude and practice regarding dengue among people in Pakse, Laos. Nagoya J Med Sci. 2009; 71:29-37. No doi 
12. Ramasamy R, Surendran SN, Jude PJ, et al. Larval development of Aedes aegypti and Aedes albopictus in peri-urban brackish water and its implications for transmission of arboviral diseases. PLOS Negl Trop Dis. 2011; 5:e1369. doi: http://doi.org/10.1371/journal.pntd.0001369

13. Comprehensive guidelines for prevention and control of dengue and dengue haemorrhagic fever. 2011; World Health Organization. Available at https://apps.who.int/iris/handle/10665/204894

14. McNaughton D, Miller ER, Tsourtos G. The importance of water typologies in lay entomologies of Aedes aegypti habitat, breeding and dengue risk: a study from northern Australia. Trop Med Infect Dis. 2018; 3:67. doi: http://doi.org/10.3390/tropicalmed3020067

15. Ramasamy R, Jude PJ, Veluppillai T, et al. Biological differences between brackish and fresh waterderived Aedes aegypti from two locations in the Jaffna peninsula of Sri Lanka and the implications for arboviral disease transmission. PLOS ONE. 2014; 9:e104977. doi: http://doi.org/10.1371/journal.pone.0104977

16. Pai HH, Lu YL, Hong YJ, et al. The differences of dengue vectors and human behavior between families with and without members having dengue fever/dengue hemorrhagic fever. Int $J$ Environ Health Res. 2005; 15:263-9. doi: http://doi.org/10.1080/09603120500155732

17. Itrat A, Khan A, Javaid S, et al. Knowledge, awareness and practices regarding dengue fever among the adult population of dengue hit cosmopolitan. PLOS ONE. 2008; 3:e2620. doi: http://doi.org/10.1371/journal.pone.0002620

18. Udayanga L, Gunathilaka N, Iqbal MCM, et al. Socio-economic, knowledge attitude practices (KAP), household related and demographic based appearance of non-dengue infected individuals in high dengue risk areas of Kandy District, Sri Lanka. BMC Infect Dis. 2018;18: 88. doi: http://doi.org/10.1186/s12879-018-2995-y

19. Ayyub M, Khazindar AM, Lubbad EH, et al. Characteristics of dengue fever in a large public hospital, Jeddah, Saudi Arabia. J Ayub Med Col Abbottabad. 2006; 18:9-13. PMID: 16977805

20. Rosenbaum J, Nathan MB, Ragoonanansingh R, et al. Community participation in dengue prevention and control: a survey of knowledge, attitudes, and practice in Trinidad and Tobago. Am J Trop Med Hyg. 1995; 53:111-7. doi: http://doi.org/10.4269/ajtmh.1995.53.111 